\title{
An easy method to reduce anterior shoulder dislocation: the Spaso technique
}

\author{
M-C Yuen, P-G Yap, Y-T Chan, W-K Tung
}

\begin{abstract}
Objective-To introduce the Spaso technique for reducing anterior shoulder dislocation by reporting the success rate of the Spaso technique performed by junior emergency medicine residents.

Design-Retrospective case series.

Setting-Urban accident and emergency department.

Participants-Patients with anterior shoulder dislocations.

Interventions-The Spaso technique was applied by the emergency medicine residents to reduce anterior shoulder dislocation.

Results-The emergency medicine residents applied the Spaso technique to reduce 16 cases of anterior dislocated shoulder during the study period. The Spaso technique was successful in 14 of 16 cases. The overall success rate was $87.5 \%$ (95\% CI 60.4, 97.8\%). No complications were noted.

Conclusion-A new method has been introduced for reduction of anterior shoulder dislocation by reporting the experience of junior residents. The Spaso technique is simple, effective and able to be performed by single operator. Although the sample size was small, the result of the study could provide background information for planning a properly designed randomised controlled trial to evaluate the Spaso technique.

(Emerg Med f 2001;18:370-372)
\end{abstract}

Keywords: shoulder dislocation; Spaso technique

There are many different methods to reduce an anterior dislocated shoulder. Different methods have their own advantages and drawbacks. ${ }^{1-3}$ Spaso Miljesic first reported the Spaso technique in 1998 in Emergency Medicine, which is a journal not listed in Index Medicus. This method has been adopted in Western Hospital in Australia for more than 15 years. According to Spaso Miljesic, this method is simple, needs minimal force, is able to be performed by a single operator and is highly effective even in inexperienced hands. ${ }^{4}$

The success rate of previously described methods in inexperienced hands was not properly evaluated. In one series the success rate of the scapular manipulation method by emergency residents was only $63 \% .^{5}$ The Spaso technique inventor claimed that this method was effective even in inexperienced hands; this aspect had not been substantiated in the article. This retrospective study was conducted to report our junior residents' initial experience of using the Spaso technique.

\section{Method}

THE SPASO TECHNIQUE

The patient is placed in the supine position; the affected arm is grasped around the wrist or distal forearm and gently lifted vertically, applying gentle traction. While maintaining vertical traction, the shoulder is slightly externally rotated (fig 1). A clunk is heard or felt when reduction is done.

If the patient experiences discomfort, they tend to lift the shoulder up off the bed. If this occurs, stop further movement of the limb but maintain traction. The pain will usually subside quickly and the patient relaxes. After a few minutes of gentle traction, reduction will usually occur. If difficulty is experienced, it may be helpful to use one hand to palpate the head of humerus and gently push it to assist reduction, while maintaining traction with the other hand. ${ }^{4}$

This study was a retrospective case series to describe the use of the Spaso technique by a group of junior residents in our unit. The Accident and Emergency Department of Kwong Wah Hospital is located in the centre of Kowloon region in Hong Kong Special Administrative Region, with a patient volume of 200000 per year. Seventeen full time emergency residents supervised by six emergency

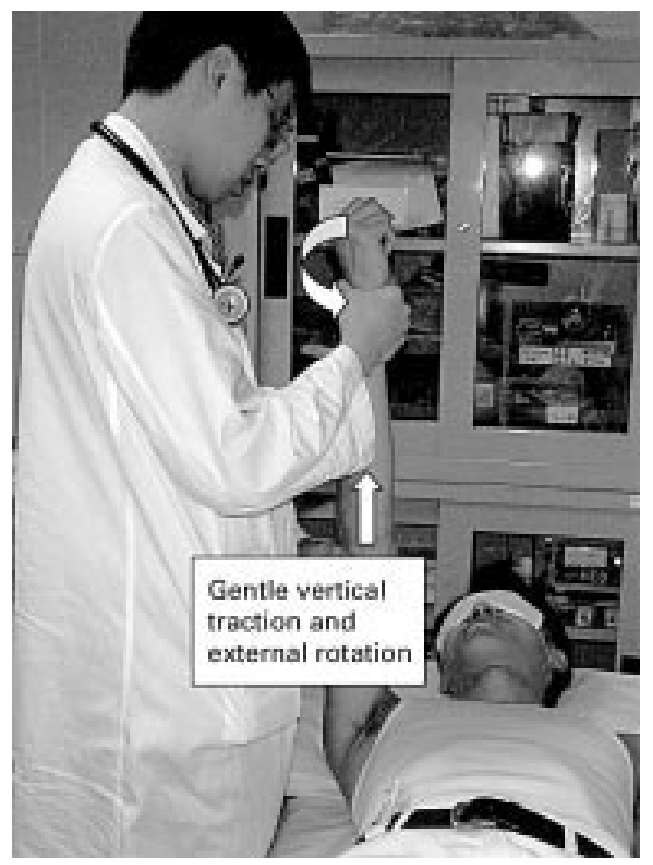

Figure 1 Vertical traction and external rotation to the affected arm. 
physicians were working in our department during the study period. Ten $(58 \%)$ of our residents have less than one year of working experience in emergency medicine during the study period. These 10 junior residents participated in the study.

The emergency medicine residents learnt the Spaso technique in a lecture conducted by the first author in June 1998. Demonstration of the Spaso technique was performed on a healthy volunteer. As the inventor of the technique did not report on the degree of sedation needed before attempting reduction, the residents were advised to use the usual sedation and analgesic available in the department titrated according to the perceived patients' discomfort. It was emphasised that they should avoid overzealous attempts and patients' comfort should be the first consideration in deciding the level of sedation needed. They were encouraged to use this method when they encountered patients with anterior shoulder dislocation.

Afterwards, a retrospective chart review of the accident and emergency department records from July 1998 to October 1998 was performed. From the record office, the records of patients seen in the study period with the diagnosis of anterior dislocated shoulder were retrieved. Patients with anterior shoulder dislocation with close reduction attempted in our department were included. Patients with anterior shoulder dislocation with fracture of humerus were excluded from analysis.

The demographic data, history of prior ipsilateral shoulder dislocation, medication given, successfulness of the attempt, and any complications developed were extracted from the record. The post-reduction radiographs were traced from the radiology department to document any iatrogenic bony fracture resulted from the reduction.

Fleiss exact formula was adopted to calculate the $95 \%$ confidence intervals of the success rate because the sample size was small.

\section{Results}

In the study period, 18 patients met the inclusion criteria for the study. The Spaso technique was applied in 16 patients (table 1). Nine $(56 \%)$ of the patients were men. The mean patient age was 48 years (range 22 to 81 ).

Table 1 Patient characteristics

\begin{tabular}{llllll}
\hline Patient & Sex & Age & $\begin{array}{l}\text { History of prior } \\
\text { ipsilateral dislocation }\end{array}$ & $\begin{array}{l}\text { Sedation and analgesic } \\
\text { used }\end{array}$ & Successful \\
\hline 1 & F & 75 & Nil & Entonox & No \\
2 & F & 25 & 3 & Entonox & Yes \\
3 & F & 69 & 2 & Entonox & Yes \\
4 & M & 61 & 1 & Entonox & Yes \\
5 & M & 32 & 2 & Entonox & Yes \\
6 & M & 24 & 2 & Valium & Yes \\
7 & M & 34 & Nil & Valium & Yes \\
8 & M & 25 & Nil & Valium & Yes \\
9 & F & 75 & Nil & Valium & Yes \\
10 & F & 55 & Nil & Valium & Yes \\
11 & M & 22 & Nil & Valium & Yes \\
12 & F & 81 & Nil & Valium & Yes \\
13 & M & 23 & 3 & Valium & Yes \\
14 & F & 81 & Nil & Valium & Yes \\
15 & M & 31 & Nil & Valium and pethidine & \\
16 & M & 61 & Nil & &
\end{tabular}

Of the dislocations reduced by the Spaso technique, the overall success rate was $87.5 \%$ (95\% CI 60.4, 97.8\%). The Spaso technique failed in two patients. One patient's dislocated shoulder could not be reduced successfully in the accident and emergency department despite the use of other methods. This patient needed close reduction under general anaesthesia by an orthopaedic surgeon. The second patient developed severe vomiting after entonox inhalation and the resident abandoned the reduction. Her dislocated shoulder was reduced by an emergency physician on duty using the Milch manoeuvre.

The medication used for reduction was at the resident's discretion. Of the successful reductions, five $(36 \%)$ patients received entonox. They all had history of prior ipsilateral shoulder dislocation. Eight $(57 \%)$ patients received intravenous valium before reduction. Of the patients who received valium before reduction, only one had history of prior shoulder dislocation. The average valium dose used was $5 \mathrm{mg}$. Only one patient received both intravenous valium and pethidine because he suffered from severe pain and cried inside the department. The resident thought it was more humane to give more sedation and analgesic to him. The type of sedation and analgesic used might reflect that our residents did follow the advice of giving the drug according to the perceived patients' discomfort.

Neither neurovascular deficit nor iatrogenic fractures were observed after the close reduction in this series.

\section{Discussion}

Spaso Miljesic and Anne-Maree Kelly first reported the Spaso technique in 1998 in Emergency Medicine. Although they concluded that the technique is simple, requires minimal force, is able to be performed by a single operator and is highly effective, their statement is not supported with any numerical evidence. ${ }^{4}$ Moreover, the degree of sedation required before attempting reduction was not reported.

The success rate of different close reduction methods for anterior shoulder dislocation reported in previous studies ranged from $70 \%-100 \% .^{6-10}$ Most of these studies were based on experienced personnel as operators except the series reported by McNamara. In McNamara's scapular manipulation technique series, the residents were only able to reduce 12 of 19 cases $(63 \%)$. The success rate of $87.5 \%$ in this series was comparable to other series performed by experienced orthopaedic surgeons and emergency physicians. The high success rate in junior residents reflected the technical ease.

The design of this study was not intended to demonstrate the superiority of the Spaso technique over previous described methods. Moreover, direct comparison of our success rate with that of previously reported methods was not reasonable as there might be racial differences in patient characteristics like body size, and the small sample size of the study also might invalidate the comparison. The main aim of this small series was to gather initial 
experience of using the Spaso technique outside the reported centre and hopefully could arouse the interest of other emergency physicians on this technique.

From the experience gained so far the Spaso technique was found to have several advantages over previously described methods.

In the Spaso technique, the steps for performing the reduction are very simple and only one operator is required. The main action to reduce the dislocated shoulder is the vertical traction and external rotation of the shoulder. Therefore, it is more straightforward than the Kocher's manoeuvre or the Milch manoeuvre. In scapular manipulation, patients are required to lie prone. If the cervical spine needed immobilisation in multiple traumatised patients, the use of scapular manipulation would be very difficult if not impossible. ${ }^{11}$ In the Spaso technique the patient is in supine position, the airway and the cervical spine would not be jeopardised.

The Spaso technique is atraumatic. Counter-traction on the axilla is not required. The risk of damaging the brachial plexus is minimal. ${ }^{12}$ Moreover, the force needed for reduction is smaller in the Spaso technique compared with the traction force required in traction and counter-traction method, as the operator could not add on their body by leaning backwards to increase the traction force.

The Spaso technique relies on sound biomechanical principles in that in the overhead position, all of the shoulder muscles course directly upwards inserting into the humerus thereby assisting reduction to the anatomical position. ${ }^{4}$ This contrasts with the methods performed with the arm at the side, where each of the shoulder muscles is running in a different direction usually requiring the use of more force or more sedation and hence the risk of fracture is increased. ${ }^{12} 13$

There were several deficiencies in this retrospective series. Firstly, not every patient with shoulder dislocation presented to the department was recruited. There was a possibility of selection bias as the residents might exclude those difficult cases. During the study period, only two cases of anterior shoulder dislocation were reduced by other methods. Therefore, the success rate would not be seriously biased. Secondly, the administration of premedication was not standardised; therefore the type of analgesic and sedation that should be used for reduction was not defined. Thirdly, the level of patient discomfort during the reduction and the mean reduction time could not be documented. In this retrospective series there was no way to report the two items. In general, reduction usually occurred within one to two minutes in this series once the vertical traction force was applied and patient would tolerate the procedure quite well. Finally, six patients had history of prior ipsilateral dislocation. There might be an argument that this group of patient biased the high success rate, as it was generally believed that reduction of a recurrent dislocation was easier than first time dislocation. To resolve the last two concerns, a properly design randomised control trial is indicated.

With the information obtained from this study, we recommend a randomised controlled trial to compare the effectiveness of the Spaso technique with the other well known methods of close reduction, and to assess the patient preference for this technique as compared with previous described methods. Only a good comparison trial will give an answer to whether the Spaso technique is an ideal method among the existing method for reduction of anterior shoulder dislocation.

There are already many methods described in the literature for reduction of anterior shoulder dislocation. ${ }^{1-3}$ The choice of a particular method depends on individual operator preference and experience. The Spaso technique is simple and atraumatic. Our resident have confirmed that this technique was easy to learn and to master. Based on the present evidence, we concluded that the high success rate of the technique among the residents would be attributed to the following properties: simple, atraumatic, biomechanical safe and single operator is required. However, literature regarding the Spaso technique is very scanty, more researches on the Spaso technique is indicated. Hopefully our report provides more information about the technique and can initiate more researches on this method.

M C Yuen would like to thank Dr Y P Li for his helpful advice in the preparation of this report. The abstract of this paper was presented at the 11th World Congress for Disaster and Emergency Medicine, Osaka, Japan, June 1999.

Contributors

$M$ C Yuen is the senior medical officer of the Accident and Emergency Department of Kwong Wah Hospital. W K Tung is the chief of services and consultant of the department. P G Yap and Y T Chan are residents of the department. W K Tung initiated the idea of writing this short report. M C Yuen designed the study, collected the data, reviewed the literature, and wrote up the report. P G Yap and Y T Chan assisted in data collection. $M$ C Yuen is the guarantor of this report.

Funding: none.

Conflicts of interest: none.

1 Gleeson AP. Anterior glenohumeral dislocation: what to do and how to do it. F Accid Emerg Med 1998;15:7-12.

2 Riebel GD, McCabe JB. Anterior shoulder dislocation: A review of reduction techniques. Am $\mathcal{F}$ Emerg $\mathrm{Med}$ 1991;9:180-8.

3 Wen DY. Current concepts in the treatment of anterior shoulder dislocations. Am f Emerg Med 1999;17:401-7.

4 Miljesic S, Kelly AM. Reduction of anterior dislocation of the shoulder: the Spaso technique. Emergency Medicine 1998;10:173-5.

5 McNamara RM. Reduction of anterior shoulder dislocations by scapular manipulation. Ann Emerg Med 1993;22: tions by

6 Kothari RU, Dronen SC. Prospective evaluation of the scapular manipulation technique in reducing anterior shoulder dislocations. Ann Emerg Med 1992;21:1349-52.

7 Mirick MJ, Clinton JE, Ruiz E. External rotation method of shoulder dislocation reduction. $f \mathrm{Am}$ Coll Emerg Phys 1979;8:528-31.

8 Leidelmeyer R. Reduced! A shoulder subtly and painlessly. F Emerg Med 1977;9:223-4

9 Beattie TF, Steedman DJ, McGowan A, et al. A comparison of the Milch and Kocher techniques for acute anterior dislocation of the shoulder. Injury 1986;17:349-52.

10 Thakur AJ, Narayan R. Painless reduction of shoulder dislocation by Kocher's method. I Bone foint Surg Br 1990;72B:524.

11 Dolye WL, Ragar T. Use of the scapular manipulation method to reduce an anterior shoulder dislocation in the supine position. Ann Emerg Med 1996;27:92-4.

12 Beeson MS. Complications of shoulder dislocation. Am $\mathcal{F}$ Emerg Med 1999;17:288-95.

3 Plummer D, Clinton J. The external rotation method for reduction of acute anterior shoulder dislocation. Emerg Med Clin North Am 1989;7:165-75. 